\title{
Des mots et des corps
}

Parole divine et orthodoxie populaire dans la Carélie du XIX siècle

of Words and Bodies. Divine Speech and Popular Orthodoxy in 19th century

Karelia

Sanoista ja kehoista. Jumalan sanat ja kansanomainen kristinusko 1800-luvun

Karjalassa

\section{Aleksi Moine}

\section{(2) OpenEdition}

\section{Journals}

Édition électronique

URL : https://journals.openedition.org/efo/7335

DOI : $10.4000 /$ efo.7335

ISSN : 2275-1947

\section{Éditeur}

INALCO

Édition imprimée

ISBN : 978-2-343-12463-6

ISSN : 0071-2051

\section{Référence électronique}

Aleksi Moine, "Des mots et des corps », Études finno-ougriennes [En ligne], 48 | 2017, mis en ligne le 19 juillet 2017, consulté le 08 juillet 2021. URL : http://journals.openedition.org/efo/7335 ; DOI : https:// doi.org/10.4000/efo.7335

Ce document a été généré automatiquement le 8 juillet 2021.

\section{(c) (7) (9)}

Études finno-ougriennes est mis à disposition selon les termes de la Licence Creative Commons Attribution - Pas d'Utilisation Commerciale 4.0 International. 


\section{Des mots et des corps}

Parole divine et orthodoxie populaire dans la Carélie du XIX siècle

of Words and Bodies. Divine Speech and Popular Orthodoxy in 19th century

Karelia

Sanoista ja kehoista. Jumalan sanat ja kansanomainen kristinusko 1800-luvun

Karjalassa

Aleksi Moine

\section{Introduction : Qu'est-ce qu'une incantation?}

1 La poésie populaire orale a joué en Finlande un rôle d'une grande importance dans la constitution et la définition d'une identité culturelle commune à la nation. Le terme même employé pour désigner la poésie populaire, kansanrunous, renvoie à la notion de kansa, qui possède à la fois le sens de " peuple » et celui de " nation ${ }^{1}$ ». C'est en rapport avec le terme finnois que j'emploie dans cet article le terme de poésie populaire, qui renvoie aux poèmes transmis par la tradition orale dans les diverses régions de Finlande.

2 C'est à partir du XviII ${ }^{\mathrm{e}}$ siècle, alors que la Finlande faisait encore partie du royaume de Suède, que l'intérêt pour les chants populaires de langue finnoise s'est éveillé2, mais il s'est développé en particulier tout au long du XIX ${ }^{e}$. Nourris des idées du romantisme et des théories politiques du philosophe allemand Johann Herder, selon lequel une nation se construit autour d'une langue et d'une culture communes, des étudiants de l'université de Turku ont entrepris des collectes de poèmes oraux à travers le territoire. Ces collectes se sont peu à peu institutionnalisées, avec la fondation en 1831 de la Société de littérature finlandaise, Suomalaisen Kirjallisuuden Seura (ci-après SKS), et elles se sont véritablement multipliées à partir de la publication par Elias Lönnrot du Kalevala, poème épique national composé par ses soins à partir des chants qu'il avait lui-même collectés et dont la première version est parue en 1835 . Étudiants et chercheurs financés par des bourses de la SKS ont sillonné le pays, surtout l'Est, c'est-àdire la région de Carélie, où les traditions de poésie orale étaient encore très riches et 
vivantes, jusqu'aux premières décennies du $\mathrm{xx}^{\mathrm{e}}$ siècle. Les textes retranscrits par les collecteurs se sont accumulés dans les archives de la SKS et ont été classés puis publiés dans les 34 volumes des Suomen Kansan Vanhat Runot, "Anciens poèmes du peuple finnois " (ci-après SKVR), entre 1908 et 1948, puis en 1995, ils sont aujourd'hui disponibles à travers une base de données en ligne ${ }^{3}$.

C'est sans doute le genre épique qui a attiré le plus l'intérêt des collecteurs et des chercheurs, notamment en raison de l'importance du Kalevala considéré comme l'équivalent finnois de l'Iliade ou de l'Odyssée. Toutefois, parmi les poèmes collectés, les chants lyriques et les incantations ne sont pas en reste et des milliers de textes ont été préservés : les SKVR contiennent plus de 30000 incantations. Pour désigner ce genre particulier de la tradition orale, les chercheurs finnois utilisent principalement le terme de loitsu qui, toutefois, comme le remarque Henni Ilomäki (2014, p. 7), n'est que très rarement employé par les informateurs eux-mêmes. Ceux-ci lui préfèrent les expressions de sanat, "les mots", luvut, "les choses lues, comptées", virsi, "parole, mot", ou "cantique", voire celui de synty, "naissance», pour désigner les incantations étiologiques qui racontent l'origine d'un phénomène ou d'un être. L'acte lui-même d'incanter est souvent caractérisé par les verbes sanata-formé sur sana, le " mot "- " enchanter ", laulaa, " chanter ", ou plutôt " enchanter, incanter " dans ce contexte. Quel que soit le terme utilisé, il semble que l'accent soit mis sur la parole, en tant qu'elle est voix et que les mots sont prononcés.

4 La définition de l'incantation que j'adopte dans cet article 4 est toutefois avant tout textuelle, puisque c'est sur des textes collectés au cours du XIX siècle et jusqu'aux premières décennies $\mathrm{du} \mathrm{xx}^{\mathrm{e}}$ que se fonde mon étude. Nous sommes, de cette manière, tributaires des recherches de nos collègues passés et de leur manière d'aborder la culture populaire. Formellement, les incantations ne se distinguent pas des autres genres poétiques publiés dans les SKVR. Ce sont des poèmes en vers kalévaléens, c'est-àdire en tétramètres trochaïques, dans lesquels les assonances et les allitérations jouent un rôle très important, de même que les parallélismes sémantiques entre des vers qui se suivent. Le contenu même des incantations peut se retrouver également dans d'autres types de poèmes: certains motifs, certains vers qui se trouvent dans des incantations sont utilisés dans des poèmes épiques, voire lyriques.

C'est en effet dans leur fonction que réside la différence principale. Comme le note Hautala (1954, p. 15), « la performance des incantations se fait toujours avec un objectif précis; on les utilise précisément et uniquement lorsque l'exigent des besoins pratiques ».

Dans les années 1950 et 1960, les recherches ont commencé à se développer autour de la fonction performative de la parole qui peut agir sur le monde, notamment autour des travaux de John Austin. Du contenu verbal, textuel, les chercheurs en sont venus à s'intéresser davantage à la notion de performance : les incantations étaient utilisées pour agir sur l'environnement et le transformer, en utilisant les divers êtres et forces qui y existent. Divers types d'incantations cohabitaient; on pouvait les utiliser pour l'amour et la fertilité, pour protéger le bétail, ou bien dans le cadre d'accouchements ou de maladies.

7 Si Elias Lönnrot a permis un développement considérable de l'intérêt pour les chants populaires, il a également été à l'origine d'un tournant dans la manière d'aborder les incantations. Les incantations ont très tôt attiré l'attention de savants comme Henrik Porthan, qui les considère comme le genre le plus finnois dans son ouvrage 
Dissertatio de Poësi Fennica, composé entre 1766 et 1778. Mais c'était principalement dans leur rapport au christianisme, à la religion et à la mythologie «païennes » finnoises, c'est-à-dire aux croyances populaires, qu'elles étaient examinées. Elias Lönnrot, à travers sa thèse de doctorat, Om finnarnes magiska medicin, «De la médecine magique des Finnois ", en 1832, a été le premier à introduire l'étude des incantations dans le cadre de la médecine populaire ${ }^{5}$ (Piela, 2010, p. 8). La pratique médicale d'Elias Lönnrot lui-même, partagée par d'autres collecteurs de chants populaires, n'a probablement pas été sans effet sur son intérêt pour les pratiques de médecine populaire, comprise comme la médecine qui se fonde sur des traditions locales et dont le praticien n'est pas reconnu par une institution officielle (université, école de médecine). Ainsi, outre les incantations, des informations ont été recueillies sur les pratiques employées pour guérir des malades. La plupart des incantations collectées sont comprises comme des incantations de guérison, utilisées dans le cadre d'un "rituel de guérison » qui est accompli par un spécialiste, le tietäjä (littéralement: "celui qui sait»), dont les connaissances permettent d'entrer en rapport avec les forces non humaines et d'agir de cette façon sur l'environnement, pour guérir le patient.

Ce rituel de guérison comprend toute une série d'éléments verbaux et non verbaux : une gestuelle, des actions particulières, un matériel spécifique comme des onguents, un cadre spatio-temporel déterminé, et la façon même de proférer l'incantation a son importance. C'est la combinaison de ces différents éléments qui permet de donner son efficacité au rituel de guérison. Malheureusement pour nous, une reconstruction détaillée des pratiques se révèle extrêmement difficile, voire impossible, dans la mesure où peu d'informations extérieures sur le contexte de performance ont été notées par les collecteurs (bien souvent, même les informations sur le contexte de collecte ne sont pas d'une grande précision). C'est donc principalement sur la sphère verbale et le contenu sémantique des incantations, considérées comme textes mais au sein d'une performance, que je me concentre.

Il convient cependant de remarquer que les textes contiennent en eux-mêmes des informations essentielles sur la pratique du tietäjä. S'ils ne permettent certainement pas une reconstruction précise des actions accomplies dans le cadre de la guérison d'un patient, il n'en reste pas moins que l'incantateur tient un discours sur ses propres pratiques. En effet, un grand nombre d'incantations contiennent des motifs métapoétiques, voire métapragmatiques, à travers lesquels le locuteur décrit ce qu'il dit et ce qu'il fait. L'un de ces motifs est particulièrement intéressant: En puhu omalla suulla, puhun suulla puhtalla, «Je ne parle pas avec ma propre bouche, je parle avec une bouche pure ". Il se trouve, avec diverses variantes, dans 160 textes collectés à travers la Finlande, dont 87 dans deux régions de Carélie, la Carélie du Nord (Pohjois-Karjala, 52 textes), et la Carélie du Ladoga (Laatokan Karjala ${ }^{6}, 35$ textes), sur lesquels je me concentrerai. Le choix de cette délimitation géographique s'explique d'une part par le fait que la plupart des textes ont été collectés dans ces régions et, d'autre part, parce que cela permet d'avoir une plus grande homogénéité sociale et culturelle. En effet, l'est de la Finlande était plus influencé par l'orthodoxie que par le catholicisme et le luthéranisme, et on y pratiquait davantage l'agriculture sur brûlis, ce qui a conduit à un réseau de villages moins dense, dans lequel la famille jouait un rôle primordial, devant garantir, dans la mesure du possible, une autosuffisance (Stark, 2006, p. 50). Chronologiquement, les textes remontant pour la plupart au XIX siècle ont été recueillis entre 1828 et 1923, la plupart l'ayant été dans les décennies 1880-1910. 
10 Mon objectif, dans cet article, est d'examiner la manière dont le pouvoir de la parole est représenté verbalement dans les incantations, en analysant le discours même que le tietäjä tient sur sa propre pratique pour la légitimer. À travers ce motif, c'est principalement la place du christianisme dans le rituel de guérison que je souhaite évaluer. Il ne s'agit pas de faire une liste exhaustive des thèmes et motifs chrétiens, mais plutôt d'observer comment ceux-ci participent à la définition de la communauté villageoise et du rituel incantatoire. En un mot, comment la pratique des incantations s'insère d'abord dans le cadre d'un christianisme populaire, qui peut ne pas correspondre aux vues de l'Église (orthodoxe) mais qui n'en utilise pas moins le vocabulaire et les concepts chrétiens, adaptés à la culture vernaculaire.

\section{Le christianisme populaire en Carélie}

11 La christianisation de la Carélie s'est faite de manière très lente et progressive, le christianisme venant se mêler aux croyances et aux pratiques vernaculaires. Les premiers témoignages archéologiques d'une présence d'objets chrétiens remontent aux $\mathrm{x}^{\mathrm{e}}$ et $\mathrm{XI}^{\mathrm{e}}$ siècles, et c'est à partir du $\mathrm{XIII}^{\mathrm{e}}$ siècle que les sources écrites, principalement les chroniques de Novgorod, évoquent la christianisation de la région : la Carélie, disputée par la Suède et Novgorod, a ainsi été soumise à la double influence du catholicisme et de l'orthodoxie. La formation du christianisme populaire en Carélie date du Xve siècle : les nouvelles doctrines se sont adaptées aux coutumes locales et ont commencé à être interprétées à travers le prisme des pratiques vernaculaires préexistantes (Laitila, 2000, p. 51-52). Il ne s'agit pas d'un simple remplacement des figures ou concepts préchrétiens par un système de croyances chrétien, mais plutôt de l'utilisation du vocabulaire chrétien pour décrire un environnement qui a gardé les caractéristiques qu'il avait auparavant dans la mesure où les structures sociales n'ont pas changé, mais qui a commencé à être perçu comme chrétien.

12 L'orthodoxie populaire s'exprimait d'abord à travers les festivités, les pratiques de vénération des saints patrons du bétail ou de la pêche, mais aussi à travers le grand nombre de légendes. La confrontation avec le christianisme officiel de l'Église n'a pas été sans heurts : il est arrivé régulièrement que l'Église s'inquiète du « paganisme » des habitants de Carélie. En témoignent par exemple les missions des moines Ilia et Nikifor au XVI ${ }^{\mathrm{e}}$ siècle ${ }^{7}$; encore en 1907, un prêtre de la paroisse de Salmi décrit dans le journal Aamun koitto les habitants de sa paroisse comme les plus ignorants et superstitieux de tout le pays (Stark, 1996, p. 143).

13 La manière d'aborder les incantations n'est pas sans lien avec l'orthodoxie populaire: la poésie kalévaléenne a longtemps été considérée en opposition avec le christianisme, dans un contexte de recherche d'indépendance nationale vis-à-vis de la Russie. Les poèmes collectés intéressaient en partie dans la mesure où ils représentaient les restes d'un paganisme préchrétien, une forme de culture carélienne "authentique ", qui se distinguait de la culture russe orthodoxe (Laitila, 2000, p. 66-71). Ne pas reconnaître l'aspect chrétien des incantations est un phénomène qui dépasse le cadre de la Finlande et de la Carélie, et se retrouve dans la recherche sur d'autres cultures également: David Elton Gay suggère que la différence entre paganisme et christianisme est une distinction qui a du sens pour le chercheur qui reconstruit l'histoire, étape par étape, de la christianisation, mais ne correspond pas nécessairement aux conceptions vernaculaires des populations qui utilisaient ces pratiques (Gay, 2009). 

chrétiens dans les textes qu'ils analysaient, et ces figures faisaient partie à part entière de typologies comme la Suomalainen mytologia, «Mythologie finnoise » de Martti Haavio (1967). Néanmoins, c'était toujours en contraste avec les figures païennes qu'elles apparaissaient ; et si le syncrétisme était reconnu, les incantations, comme le reste de la poésie orale, ont souvent servi à la reconstruction d'une mythologie spécifique.

C'est donc dans une tentative de prendre en compte le point de vue émique que je souhaite aborder les incantations à travers le concept de christianisme populaire. Cette approche s'insère dans le cadre des études de folklore contemporaines, qui insistent sur l'importance primordiale de la contextualisation, sans laquelle le texte perd son sens. Le texte, et cela est valable à la fois pour les incantations et pour les autres genres de la poésie populaire, ne peut se comprendre, en effet, que comme performance, dans un contexte historique, culturel, social, donné, avec lequel il entre en dialogue constant, pour créer du sens (Tarkka, 2005, p. 12, 54-56). La région étudiée dans le présent article est globalement chrétienne, influencée par l'orthodoxie, mais il existe des différences importantes entre les pratiques locales, que cet article n'a pas la prétention d'examiner en détail. Le motif étudié a une vaste distribution géographique et peut, par conséquent, servir de dénominateur commun à différents territoires, qui partagent des éléments culturels communs. L'objectif est donc d'évaluer, à travers un motif métapoétique, la manière dont le tietäjä représente l'efficacité de la parole divine dans le rituel de guérison.

\section{La délimitation chrétienne du cadre spatio-temporel}

\section{L'Ici chrétien, le Là-bas non chrétien}

16 Une incantation fonctionne comme un moyen de communication entre le guérisseur et les êtres non humains qu'il doit manipuler pour obtenir un effet sur l'environnement, c'est-à-dire sur la maladie du patient. Chaque incantation entre donc dans un cadre spatio-temporel déterminé et construit une situation d'énonciation particulière. Plusieurs éléments entrent dans la définition de ce cadre. Le guérisseur ne s'adresse en général pas au patient, qui est cependant présent, mais uniquement aux forces non humaines, soit pour obtenir leur aide, soit au contraire pour les conjurer, selon leur nature respective; dans chacun des cas, les stratégies discursives de l'incantateur ne sont pas les mêmes. Parmi les forces adjuvantes, on compte bon nombre de figures proprement chrétiennes, comme Dieu, Jésus, la Vierge Marie, divers saints, dont les caractéristiques et les dénominations se mêlent souvent à des figures qui ont précédé le christianisme, à l'instar d'Ukko ou de Väinämöinen. Il semble toutefois que le vocabulaire chrétien soit au fondement des délimitations du cadre spatio-temporel.

Le rituel a en effet toujours lieu dans une situation bien précise et définie, un hic et nunc, qu'il convient de désigner. Lorsqu'il s'adresse aux forces adjuvantes, l'incantateur met la plupart du temps l'accent sur sa propre faiblesse, et sur celle du patient, pour indiquer l'urgence qu'il y a à trouver de l'aide. C'est ainsi le cas dans l'incantation suivante :

\begin{tabular}{|l|l}
\hline Neitsut Maaria maine(!), $\quad$ La Vierge Marie, femme,
\end{tabular} 


\begin{tabular}{|l|l|}
\hline Puhas muori on muovollinen. & Vieille femme pure, est belle. \\
\hline Tules tänne tarvitaan & Viens ici, où l'on a besoin de toi, \\
\hline Tuskahisen tupaan, & Dans la chambre où l'on souffre, \\
\hline I hätähisen huonehes, & Et la pièce où l'on a mal, \\
\hline Päästämään, pellastamaan & Pour libérer, pour sauver \\
\hline Oikie ristitty henkie ${ }^{8}$. & L'esprit qui a bien été baptisé. \\
\hline
\end{tabular}

Le tietäjä s'adresse à la Vierge Marie en l'appelant par son nom : cette adresse est un élément nécessaire qui permet d'attirer l'attention de l'être dont on demande l'aide (c'est la même stratégie qui est utilisée dans les cas de conjuration également). L'impératif est utilisé pour la faire venir dans la pièce où a lieu le rituel, pour la rendre présente. Cette pièce est caractérisée dans l'incantation non par des caractéristiques physiques mais par l'état de souffrance du patient, à travers les adjectifs tuskahinen, "où l'on souffre " et hätähinen, "où l'on a mal». Ce qui est particulièrement intéressant est cependant la façon dont le guérisseur définit le patient qui doit être guéri par la Vierge Marie : il s'agit d'oikie ristitty henkie, un «esprit qui a été bien baptisé, de façon juste, droite». Cette définition se rapproche d'une tendance des langues fenniques à désigner l'être humain selon le rapport au christianisme : ainsi, en vepse, c'est le terme de ristitu, littéralement "baptisé », " chrétien », qui est utilisé de façon générale pour l'être humain.

19 Une désignation analogue du patient se retrouve dans une autre incantation, longue de 129 vers, collectée dans la paroisse de Salmi, destinée à être prononcée lorsque l'on va au bain ; le sauna est en effet le lieu privilégié des pratiques de guérison'. En voici le début, qui consiste en une invocation de différents êtres adjuvants :

\begin{tabular}{|l|l|}
\hline Avoa, Luoja, taivas, & Ouvre, Créateur, le ciel, \\
\hline Astu alah taivahas; & Viens, descends du ciel ; \\
\hline Tule miulle avukse, armokse, & Viens-moi en aide, en miséricorde, \\
\hline Väikse, voimakse, & En force, en puissance, \\
\hline Tätä risti-kansoa päästämään, pelvastamaan! & Pour libérer, pour sauver ce peuple de la croix! \\
\hline Taivahaset anhelit, & Anges célestes, \\
\hline Tulkoa avukse, armokse, & Venez en aide, en miséricorde, \\
\hline Väikse, voimakse. & En force, en puissance. \\
\hline Sorok svätei, sorok muuzenih, & Quarante saints, quarante hommes, \\
\hline Zem deźat anhel, & Soixante-dix anges, \\
\hline
\end{tabular}




\begin{tabular}{|c|c|}
\hline 12 apostolaa, & Douze apôtres, \\
\hline Tulkoa avukse armokse, & Venez en aide en miséricorde, \\
\hline Väikse, voimakse, & En force, en puissance, \\
\hline Tätä risti-kansoa päästämään, pelvastamaan! & Pour libérer, pour sauver ce peuple de la croix! \\
\hline Se kutsuupi & Il appelle \\
\hline Solomani boabuska Kristos vooditša, & Salomon l'ancêtre, le Christ conducteur, \\
\hline Pyhän Spaasan paapoi, & Il prie le Saint Sauveur, \\
\hline Pyhän Rastavan paapoi; & Il prie la Sainte Naissance, \\
\hline Neitsut Maari[a] emonen, & La Vierge Marie, petite mère, \\
\hline Puhas [muori, muoto kaunis], & Pure [femme, belle forme], \\
\hline Tules tänne, tarvitaan, & Viens ici, on a besoin, \\
\hline Rauhutta rakentamaan, & Pour construire la paix, \\
\hline Tervehyttä tälle ristikansalle, & La santé à ce peuple de la croix \\
\hline Päästä ja pelvasta, & Libère et sauve, \\
\hline Puhu i puhtasta, & Parle et purifie, \\
\hline Vein [isändät] j.n.e. & Avec l'eau [les pères] etc. \\
\hline Keskellä kivuttomaksi, & Au milieu contre les douleurs, \\
\hline Alla aivan terveeksi, & En dessous pour la santé, \\
\hline Päällä nuurumattomaksi, & Au-dessus pour enlever les souffrances, \\
\hline Kuin omas poikas päästit ja pelvastit, & Comme tu as libéré et sauvé ton propre fils, \\
\hline Muga tämä risti-kansa päästä ja pelvasta]. & Libère et sauve ce peuple de la croix. \\
\hline Oi sie katehellinen Kabeli, & Oh toi, envieux Kabeli, \\
\hline Riekka reiän juurellinen, & Riekka des racines, du trou, \\
\hline Paku kun leppänen paalikku, & Paku comme un bout de bois d'aulne \\
\hline Risti-kansoa rikkomas, & Qui est en train de briser le peuple de la croix, \\
\hline Kastettuu kaatamas; & De faire tomber le baptisé ; \\
\hline Ei ole ristikansas rikottavaa, & Il n'y a pas à briser le peuple de la croix, \\
\hline
\end{tabular}




\begin{tabular}{|l|l|}
\hline Ei kastetus kaattavaa. & à faire tomber le baptisé. \\
\hline Saa tila, muuta maja, & Prends ta place, change de maison, \\
\hline Ennen päivän nousemista, & Avant le lever du jour, \\
\hline Auringon ylenemistä; & Le réveil du soleil ; \\
\hline Mene itseh isäntään, & Va chez ton propre père, \\
\hline Omahas emäntään ${ }^{10}$. & Chez ta propre mère. \\
\hline
\end{tabular}

L'incantateur appelle les figures adjuvantes et les invite à "venir " sur place, à se rendre présentes, en s'adressant directement à elles, qu'il s'agisse de Dieu, Jésus, Marie, des anges, des apôtres ou de divers saints. L'importance de nommer les figures adjuvantes pour les faire venir a été remarquée par Jouko Hautala, selon lequel les mots ont la caractéristique de non seulement renvoyer à une chose, de la désigner, mais aussi d'être la chose même : prononcer le nom permet de rendre la force ou la figure présente (Hautala, 1960, p. 15-16). Si l'on peut ne pas suivre toute l'argumentation de Jouko Hautala, qui considère différents stades de l'évolution de la pensée en partant d'un stade " primitif ", il n'en reste pas moins que nommer les figures qui doivent aider ou qu'il faut chasser est une des stratégies discursives utilisées le plus fréquemment dans les incantations. Il s'agit en effet d'attirer l'attention vers l'endroit où a lieu le rituel, ce qui se combine avec l'emploi de nombreux déictiques : le lieu est désigné par l'adverbe tänne, «ici » (avec mouvement), de même que la personne à guérir, tämä risti-kansa, «ce peuple de la croix ». La croix, symbole chrétien par excellence, sert ainsi de fondement à l'identité du patient qui doit être guéri : le malade apparait ici comme le représentant de la communauté villageoise, qui est caractérisée par la foi chrétienne. En lui s'incarne l'ensemble de la communauté chrétienne, et c'est de son côté que se place l'incantateur. Plus tard dans le texte, le patient est en effet nommé à plusieurs reprises ristitty, « le baptisé (qui est sous le signe de la croix)», ou kastettu, «le baptisé (en tant qu'il a été touché par l'eau bénite)». Il est intéressant de noter que c'est au moment de la lutte contre la maladie, de la conjuration du kabeli, ici, qu'intervient cette définition du patient comme baptisé. Le tietäjä nie les conséquences négatives de la maladie qui est en train de briser le baptisé : le mal n'a rien à faire à cet endroit et on lui intime de repartir chez lui, auprès de ses parents. Anna-Leena Siikala rapproche cette idée du chamanisme des peuples de Sibérie: le chaman se devait de faire un voyage vers l'au-delà, vers l'autre monde, pour obtenir une connaissance des origines du mal. Communiquer avec les parents, les ancêtres du mal, permettait d'obtenir le pouvoir sur lui dans la mesure où ceux-ci avaient le pouvoir dans leur famille (Siikala, 1999, p. 78-81). Si l'origine de ce motif est sans doute à ramener à une culture commune aux peuples de chasseurs de l'Arctique et du Nord, il n'est pas impossible d'y voir également une référence aux structures sociales contemporaines en Carélie. Dans la société rurale agricole du $\mathrm{XIX}^{\mathrm{e}}$ siècle, la famille joue un rôle primordial pour la subsistance et la survie de ses membres, elle est le fondement même de la société. Ainsi, le maître de maison, l'isäntä, est responsable de la survie de tous les membres de la famille. La maladie appartient à un monde analogue, à un "contre-monde», un vastamaailma (le concept étant développé par Ulla Piela [2010, p. 44] en opposition à 
l'idée de «ce monde ", tämä maailma, qui est le monde dans lequel vivent le guérisseur et le patient), dans lequel existent des structures sociales similaires ; l'être responsable du mal est donc soumis à l'autorité de ses parents.

\section{Le baptême comme élément clé}

21 Un élément essentiel dans cette distinction entre les deux mondes est la place du baptême. L'incantateur se place du côté des baptisés, oikie ristitty henki, « l'esprit baptisé comme il faut», par exemple, ou ristikansa, "le peuple de la croix», comme nous l'avons déjà vu. Il semble donc que le baptême vaut comme définition de la communauté, dans le cadre du rituel de guérison. Il est possible d'interpréter de cette manière un élément qu'on retrouve dans un certain nombre d'incantations, lorsque l'incantateur fait le récit du mythe étiologique des maladies. C'est un mythe très répandu, selon lequel une femme, qui peut porter plusieurs noms, comme Loviatar, Lohetar, Äkäätär ${ }^{11}$, donne naissance à neuf garçons. Après leur naissance, elle cherche à les faire baptiser, mais ne trouve personne et doit donc le faire elle-même :

\begin{tabular}{|l|l|}
\hline Ehtipä hiän ristijee, & Elle se mit à la recherche d'un baptiste, \\
\hline Vuan ei suanunnaak. & Mais n'en trouva pas. \\
\hline Ihe risti poikijaan: & Elle baptisa elle-même ses fils : \\
\hline Minkä ruaniks rapas, & L'un fut frappé du nom de raani, \\
\hline [Minkä] riijeks ritusti ${ }^{12} . .$. & Un autre, du nom de koista... \\
\hline
\end{tabular}

L'origine des maladies se comprend ainsi par analogie à la naissance des enfants ; elles apparaissent lorsque, mises au monde, elles reçoivent leur nom, leur identité. C'est le terme chrétien de ristiä, « baptiser », qui est utilisé dans l'incantation, mais il s'agit d'un baptême inversé : les fils de Loviatar reçoivent un nom qui n'est pas chrétien. C'est pour cette raison qu'ils peuvent présenter un danger pour les humains et qu'il convient de les conjurer chez eux, dans le monde qui est le leur. La suite de l'incantation citée plus haut montre également que l'église, lieu sacré du christianisme, joue un rôle important dans cette conjuration :

\begin{tabular}{|l|l|}
\hline Van tuone mie siun manoon: & Mais c'est là-bas que je te conjure : \\
\hline Kirkon kirjatun tyköhön, & Vers l'église aux écritures, \\
\hline Portin mualatun povehen; & Vers la porte aux peintures ; \\
\hline Siel on muutkin murhamiehet, & Là sont aussi les autres méchants, \\
\hline Ikuiset pahan tekiit. & Les éternels faiseurs de maux. \\
\hline Tuone vielä siun mano「aln: & Là-bas aussi je te conjure : \\
\hline
\end{tabular}




\begin{tabular}{|l|l|}
\hline Pohjan lietohon merehen, & Dans la mer du nord/du fond, \\
\hline Johon puut tyvin putoovi, & Où les arbres tombent, \\
\hline Hongat latvon lankeili. & Les troncs de la cime chutent. \\
\hline
\end{tabular}

23 Parmi les différents endroits où la maladie est renvoyée, qui sont la plupart du temps définis par leur étrangeté, leur distance par rapport au malade, au-delà des mers et des montagnes, se trouve l'église, ce qui est un phénomène relativement rare. Cela montre toutefois que l'église permet d'aider dans la bataille contre la maladie; elle peut jouer le rôle de charnière entre le tänne, l'ici où viennent les figures adjuvantes, et le tuonne, le là-bas où il faut conjurer les forces responsables du mal.

Ainsi, lorsqu'une maladie survient à un être humain, ce n'est pas parce qu'il existe un mal par nature, mais plutôt qu'il s'est produit un déséquilibre : l'être - ou la force responsable de la maladie - s'est introduit dans un corps humain, chrétien, et ne se trouve pas à la bonne place. Les deux mondes coexistent en parallèle et se ressemblent. Un des objectifs du rituel de guérison est de rétablir l'équilibre perdu et de renvoyer à sa place l'être responsable du mal, dans la société à laquelle il appartient, là où son père et sa mère l'attendent, et de ne pas le laisser dans le corps du malade.

Pendant la performance du rituel de guérison, l'incantateur qui prononce le charme destiné à guérir le patient crée ainsi une situation d'énonciation particulière, dans laquelle les personnages adjuvants, bien souvent des figures chrétiennes, apparaissent sous ses yeux et réalisent les actions. L'incantateur va même plus loin dans la description de la situation de la performance : il prend de la distance vis-à-vis de luimême et décrit ses propres actions.

\section{Incantation et présence divine : la description du rituel}

\section{Dieu comme incantateur}

26 Comme évoqué plus haut, l'incantateur tient un discours métapoétique et métapragmatique dans ses incantations, et invoque la force divine pour parler de ses propres actions. C'est ce que Lotte Tarkka appelle le "processus d'autorisation ", autorisaatioprosessi (Tarkka, 2005, p. 92-95). Ce processus, qui donne de l'autorité à l'incantation et à l'incantateur, entre en rapport avec la problématique de la croyance ou non en l'efficacité des incantations. C'est un problème qui intéressait probablement davantage les chercheurs que ceux qui étaient concernés par les pratiques, puisque ces derniers n'explicitaient pas nécessairement leur «croyance» en l'efficacité des pratiques de guérison. Cependant, les textes eux-mêmes fonctionnent comme des garanties de ce pouvoir : outre l'autorité de la tradition, celle de Dieu est prévalente ${ }^{13}$.

Le tietäjä, de façon paradoxale, se place en position de faiblesse pour garantir sa propre force et l'efficacité du rituel qu'il performe : non seulement ses propos, mais aussi ses actes viennent de Dieu lui-même. Il s'agit presque d'une identification, le guérisseur endosse « un rôle rituel » (Tarkka, 2005, p. 94): 


\begin{tabular}{|l|l|}
\hline En puhu omalla suulla, & Je ne parle pas avec ma propre bouche, \\
\hline Puhun suulla Luojan suulla; & Je parle avec la bouche, la bouche du Créateur ; \\
\hline Itše Luoja on loitšemass, & C'est le Créateur lui-même qui incante, \\
\hline Pyhä mies pelastamass $^{14}$. & Le saint homme qui sauve. \\
\hline
\end{tabular}

Dans ces vers, l'emploi du présent progressif rend le Créateur présent : il fait partie de la scène et prend la place du guérisseur qui ne parle plus avec sa bouche. Les mots qui sortent d'entre ses lèvres sont des mots divins et l'efficacité de la guérison dépend de la force divine qui est en présence.

Cette façon de devenir autre pendant le rituel de guérison est à comprendre dans les termes de l'état d'extase que devait atteindre le tietäjä, en finnois, haltioituminen. C'est ainsi qu'Elias Lönnrot déjà décrit l'extase physique :

Il existe de nombreuses variantes des paroles préparatoires (alkusanat, "paroles d'introduction ») qu'on prononçait au début. On considérait qu'elles conduisaient l'incantateur à l'état d'extase qu'il recherchait. Dans ces mots, l'incantateur parle de la force qu'il a reçue de Dieu, il flatte sa force, son art, etc. Et il se retrouve même véritablement dans un état d'extase, un état qu'on appelle en finnois olla innoissaan ou haltioissaan. Il se comporte comme un enragé, la profération des paroles devient plus violente et emportée, sa bouche s'emplit d'écume, ses dents se serrent, ses cheveux se dressent, ses yeux roulent, ses sourcils se froncent, il crache, son corps se tord, il agite les jambes, saute sur le sol et fait beaucoup d'autres gestes.

(Lönnrot, 1832, p. 10-11)

Le motif «Je ne parle pas avec ma bouche » peut ainsi être compris comme l'une des paroles d'introduction qui enclenchent l'état d'extase ; cependant, il ne fonctionne pas uniquement comme introduction puisque sa place dans les textes collectés est variable. Il reste une garantie de l'extériorité et de la supériorité du pouvoir du tietäjä, qui s'étend de la sphère verbale au reste de son corps.

31 Le guérisseur crée ainsi une représentation particulière du «je » agissant. Dans la poésie orale lyrique, le «moi » est souvent fluctuant et peut endosser divers rôles au sein d'un même texte, selon les stratégies adoptées par le chanteur (Timonen, 2004, p. 396-399). Le même phénomène se produit dans le cas des incantations, qui ont la même forme mais dont la fonction diffère : l'état d'extase est ainsi représenté par cette absence, momentanée, du « je », remplacé par Dieu, qui prend possession de son corps :

\begin{tabular}{|l|l|}
\hline Ej minussa lienekähän & Mais il n'y a rien en moi, \\
\hline omin voiminni mitänä, & Rien dans mes propres forces, \\
\hline tehtä Luojan tuttaville, & Pour agir pour les proches du Créateur, \\
\hline jalon lapsille Jumalan. & Pour les enfants du noble Dieu. \\
\hline En koske omin käsin, & Je ne touche pas avec mes propres mains, \\
\hline
\end{tabular}




\begin{tabular}{|l|l|}
\hline kosken Jiesuksen kätösin; & Je touche avec les petites mains de Jésus ; \\
\hline \multicolumn{2}{|l|}{} \\
\hline soveljammat Luojan sormet, & Les mains du Créateur appliquent, \\
\hline Jeesuksen käet käviämmät. & Les mains de Jésus passent. \\
\hline En puhu omalla suulla, etc. ${ }^{15}$ & Je ne parle pas avec ma propre bouche, etc. \\
\hline
\end{tabular}

Dans cet exemple, le «je» de l'incantateur n'existe que par la négative, à travers sa faiblesse, ou son absence de forces. Le guérisseur tient un discours à la troisième personne, comme s'il observait ses mains et ses doigts agir sans en être consciemment à l'origine. Les mains deviennent sujet, et c'est le corps physique qui remplace le "je » conscient dans les actes accomplis au cours du rituel. Du plan verbal, on passe au plan physique : les doigts ont un rôle essentiel dans la mesure où ils sont l'intermédiaire entre les deux corps, celui du guérisseur et celui du patient, et que c'est à travers le toucher que peut se réaliser la guérison du malade.

\section{Deux corps en présence : la réalité physique et symbolique}

Ulla Piela estime que le toucher du guérisseur est une des parties essentielles du rituel de guérison. Il permet d'établir un contact physique avec le malade, un dialogue corporel et une proximité rituelle, lorsque par ses mots un " espace rituel » a été créé. Le toucher est vécu comme un geste de guérison et permet de rattacher le malade au monde physique, à ce monde, en renvoyant la maladie dans le contre-monde ; il s'agit d'un « toucher rituel» (Piela, 2005, p. 26-27).

Pendant le rituel, la barrière entre les mondes physique et symbolique s'efface au gré du guérisseur (Ilomäki, 2014, p. 42). Celui-ci devient Dieu, ou Jésus en tant que Dieu, tandis que le patient devient Jésus en tant qu'il renaît après sa mort, oint par les soins de Marie. En effet, l'utilisation d'onguents comme intermédiaires entre les deux corps est très fréquente. Souvent, ce qui importe est moins la composition physique des onguents, qui peuvent être faits à partir d'ingrédients tout à fait quotidiens trouvés dans la maison, que la charge verbale qu'ils contiennent, parce qu'on les a incantés, qu'on a prononcé un charme en eux. Ainsi, les onguents jouent le rôle de transfert de pouvoir depuis le tietäjä jusqu'au patient. Ils sont souvent décrits comme des fluides corporels divins : le lait de Marie, le sang de Jésus, la sueur et les larmes du Créateur ${ }^{16}$. Et c'est bien souvent Marie qui oint le malade, comme dans l'exemple suivant:

\begin{tabular}{|l|l|}
\hline Neitsyt Maaria emonen, & Vierge Marie, petite mère, \\
\hline Rakas äiti armollinen, & Chère mère miséricordieuse, \\
\hline Pistä suluka sulaan voihen, & Trempe la plume dans le doux onguent, \\
\hline Voija alta, voija piältä, & Oins par-dessous, oins par-dessus, \\
\hline Kerta keskii sipase, & Enduis aussi au milieu, \\
\hline
\end{tabular}




\begin{tabular}{|l|l|}
\hline Alta nuurumattomaksi, & En dessous pour enlever les douleurs, \\
\hline Piältä tuntumattomaksi, & Par-dessus pour qu'il ne sente plus, \\
\hline Jotta saisi sairas muata, & Pour que puisse se reposer le malade, \\
\hline Vois voihkava levätä & Que puisse être tranquille le souffrant, \\
\hline Minun silimin nähtyveni, & Alors que mes yeux regardent, \\
\hline Suin sulin puheltueni; & Alors que ma bouche parle; \\
\hline Enkä puhu omalla suulla, & Et je ne parle pas avec ma propre bouche, \\
\hline Puhun suulla puhtahalla, & Je parle avec la bouche pure, \\
\hline Läiköttelen lämpösellä, & Je répands la chaleur, \\
\hline Herran hengellä hyvällä17. & Avec le bon esprit du Seigneur. \\
\hline
\end{tabular}

Deux vers sont particulièrement intéressants dans ce texte : la présence et l'action de Marie sont attestées par le témoignage du guérisseur qui observe la Vierge agir, de ses propres yeux. Le corpus ne contient pas d'identification du guérisseur avec Marie au travers de la première personne, comme cela arrive avec Dieu et Jésus; toutefois, c'est bien elle qui agit ici et prend la place du tietäjä qui enduit le corps du malade à l'aide d'une plume.

Cette confusion des identités, cette superposition de différents rôles sont à rapprocher d'instances où le guérisseur parle à travers la voix même de Jésus :

\begin{tabular}{|l|l|}
\hline Toipa noita voiteita. & Et [Mehiläinen] apporta ces onguents. \\
\hline "Opi Jeesus kielelläsi, & « Apprends, Jésus, avec ta langue, \\
\hline Onko nämä niitä voiteita, & Si ce sont bien ces onguents, \\
\hline Millä on itsees voijettiin?” & Avec lesquels on t'a oint toi-même? \\
\hline Toipa (noita voiteita), & Et il apporta ces onguents, \\
\hline Oppi Jesus (kielelläsi(?)): & Jésus apprit avec sa langue : \\
\hline "Nämä (on niitä voiteita), & «Ce sont bien ces onguents, \\
\hline Millä on minua (voijettiin).” & Avec lesquels on m’a oint moi-même. » \\
\hline Voia oalla, voia päällä, & Oins par-dessous, oins par-dessus, \\
\hline
\end{tabular}




\begin{tabular}{|l|l|}
\hline Voia kerta keskieh & Oins également au milieu, \\
\hline Siivellä simasella, & Avec ton aile d'hydromel, \\
\hline Sullalla hopeisella!18 & Avec ta plume d'argent! \\
\hline
\end{tabular}

37 Dans ces quelques vers sont caractérisés les onguents utilisés dans le rituel de guérison; le guérisseur cherche à affirmer l'origine divine, de l'autre monde, des onguents (Piela, 2007), et, pour ce faire, après avoir décrit le voyage de Mehiläinen vers le monde au-delà des sept mers pour trouver les substances dont il aurait besoin, il utilise la voix de Jésus. Le dialogue entre le guérisseur et Jésus permet une identification complète : les mêmes vers sont répétés, d'abord sous forme de question, puis sous forme de réponse, et, par l'emploi du discours direct, l'incantateur adopte le « je » de Jésus pour un instant.

Ainsi, dans l'espace du rituel, séparé de la vie quotidienne, les relations entre le guérisseur et le patient acquièrent un nouveau sens; leur statut se transforme. Le toucher qui unit leurs corps est une répétition du toucher qui unit les doigts de Marie au corps blessé de Jésus, et cette superposition des rôles, cette identification avec des figures divines, est une condition de l'efficacité de l'incantation comme pratique de guérison.

\section{Conclusion}

39 L'analyse des motifs métapoétiques contenus dans les incantations finnoises de Carélie permet de mettre en lumière la conception particulière du pouvoir de la parole telle qu'elle est représentée dans les textes. Les mots n'ont pas un pouvoir en eux-mêmes : l'efficacité de la parole réside principalement dans le fait qu'elle permet de constituer un cadre spécifique, distinct de la vie quotidienne, dans lequel divers niveaux de réalité, physique et symbolique, se mêlent et se confondent. Dans cet espace rituel spécifique, les acteurs du rituel et les figures divines appelées à l'aide se superposent : le tietäjä devient Dieu, Jésus, ou agit comme Marie, et le patient devient Jésus qui se fait oindre. Le Christ occupe ainsi deux positions différentes mais non contradictoires, en tant que Dieu, d'une part, en tant qu'homme, de l'autre.

Il s'agit donc d'une pratique éminemment chrétienne bien que ses origines ne soient pas à chercher dans l'Église. Les figures chrétiennes se sont adaptées aux pratiques vernaculaires pour former un christianisme populaire, qui ne correspond pas nécessairement aux vues de l'Église officielle mais qui n'en est pas moins chrétien pour autant. Le vocabulaire chrétien, les conceptions chrétiennes occupent une place importante dans les textes collectés et dans la définition du rituel.

41 Si l'origine du pouvoir du tietäjä semble être divine, le guérisseur n'est toutefois pas omnipotent, comme le montre clairement une remarque qui suit l'incantation VII $_{4}$ loitsut 1740 : Kun ei tulla kuolendaksi, niin se pääsöö, kun lienöö kovin Jumala kironnu, niin äsken se ei pääse. «Lorsqu'on ne meurt pas, alors elle fonctionne, mais si on a juré fort contre Dieu, alors elle ne libère pas. » Le pouvoir de guérison est donc limité au caractère de bon chrétien du patient et à la volonté de Dieu, ce qui permet de mettre hors de cause le guérisseur en cas d'échec. 


\section{BIBLIOGRAPHIE}

GAY David Elton, 2009, “On the Christianity of Incantations”, in ROPER Jonathan (ed.), Charms, Charmers and Charming; International Research on Verbal Magic, Basingstoke: Palgrave Macmillan. HAAVIO Martti, 1967, Suomalainen mytologia, WSOY, Porvoo.

HAKAMIES Pekka, 1983, "Sauna ja kansanomainen terveydenhoito", in LAAKSONEN Pekka \& PIELA Ulla (eds.), Kansa parantaa, Helsinki: Suomalaisen Kirjallisuuden Seura.

HAUTALA Jouko, 1954, Suomalainen kansanrunoudentutkimus, Turku: Suomalaisen Kirjallisuuden Seura.

HAUTALA Jouko, 1960, "Sanan mahti", in KеKO Jumin, Tutkielmia kansanrunoustieteen alalta, Helsinki: Suomalaisen Kirjallisuuden Seura.

ILOMÄKI Henni, 2014, Loitsun mahti, Helsinki: Suomalaisen Kirjallisuuden Seura.

LAAKSONEN Pekka, 1983, "Kansanlääkinnän tutkimuksen vaiheita", in LAAKSONEN Pekka \& PIELA Ulla (eds.), Kansa parantaa, Helsinki: Suomalaisen Kirjallisuuden Seura.

LAITILA Teuvo, 2000, "Popular Orthodoxy, Official Church and State in Finnish Border Karelia before World War II", Folklore 14, pp. 49-74.

LÖNNROT Elias, 1832, Om finnarnes magiska medicin, Helsinki.

PIELA Ulla, 2005, "Parantajan rituaalinen kosketus", in HUTtUnEn Sirpa \& NUOLIJÄRVI Pirkko (ed.), Tahdon sanoa. Kirjoituksia kielen ja perinteen voimasta, Helsinki: Suomalaisen Kirjallisuuden Seura. PIELA Ulla, 2007, "Parantajan sanat ja taivaalliseet voiteet", Duodecim, 122, pp. 2880-2888.

PIELA Ulla, 2010, Kansanparannuksen kerrotut merkitykset Pohjois-Karjalassa 1800-ja 1900-luvuilla, Joensuu: Publications of the University of Eastern Finland.

SIIKALA Anna-Leena, 1999, Suomalainen šamanismi, Helsinki: Suomalaisen Kirjallisuuden Seura.

SKVR = Suomen Kansan Vanhat Runot, 1908-1948, 1995, Helsinki: Suomalaisen Kirjallisuuden Seura . STARK Laura, 1996, “The Folk Interpretation of Orthodox Religion in Karelia from an Anthropological Perspective", Studies in Folklore and Popular Religion, 1, pp. 143-157.

STARK Laura, 2006, The Magical Self: Body, Society and the Supernatural in Early Modern Rural Finland, Helsinki: Suomalainen Tiedeakatemia.

TARKKA Lotte, 2005, Rajarahvaan laulu. Tutkimus Vuokkiniemen kalevalamittaisesta runokulttuurista 1821-1921, Helsinki: Suomalaisen Kirjallisuuden Seura.

TIMONEN Senni, 2004, Minä, tila, tunne. Näkökulmia kalevalamittaiseen kansanlyriikkaan, Helsinki: Suomalaisen Kirjallisuuden Seura.

VÄSTRIK Ergo-Hart, 2010, « Les pratiques superstitieuses des habitants de la pjatina vote dans la première moitié du XVI ${ }^{\mathrm{e}}$ siècle », Études finno-ougriennes, 42, p. 35-82.

\section{NOTES}

1. Le mot nation est pris ici au sens herdérien (N.d.R.). 
2. Pour une histoire détaillée des débuts des études de folklore en Finlande, en particulier de la poésie orale de langue finnoise, jusqu'aux années 1950, consulter l'étude de Hautala (1954).

3. Cette base de données, qui a été utilisée pour rassembler les matériaux de l'article, est accessible à l'adresse suivante : www.skvr.fi [consulté le 28.03.2017].

4. Cet article se fonde sur les recherches faites pour un mémoire de master 2 recherche soutenu en juin 2015 à l'Inalco sous la direction d'Eva Toulouze, " "Je ne parle pas avec ma propre bouche" : la représentation du pouvoir de la parole dans les incantations finnoises de Carélie ».

5. Pour une synthèse de l'histoire de la recherche sur la médecine populaire en Finlande, voir Laaksonen, 1983, et Piela, 2010.

6. Aussi appelée Raja-Karjala, Carélie frontalière.

7. Pour une interprétation détaillée des sources sur ces missions dans le cadre du christianisme populaire, voir Västrik, 2010.

8. SKVR VII loitsut 1479.

9. Voir par exemple Hakamies, 1983.

10. SKVR VII 4 loitsut 1740.

11. En général caractérisée comme la fille de Tuoni, le maître du monde des morts (Tuonela), ou bien rapprochée de Louhi, la maîtresse de Pohjola, elle est dans tous les cas associée au froid, à l'obscurité, au nord ou au monde des morts.

12. SKVR VII 4 loitsut 2828.

13. Il est cependant tout à fait possible qu'il ait existé une forme d'incantations « noires » faisant appel aux forces démoniaques, au diable, mais que les informateurs n'aient pas désiré les communiquer aux collecteurs (Tarkka, 2005, p. 93).

14. SKVR VII 3 loitsut 1035.

15. SKVR VII 4 loitsut 3031.

16. Voir par exemple SKVR VII loitsut 790 ou VII 4 loitsut 2551 .

17. SKVR VII 4 loitsut 2828.

18. SKVR VII 3 loitsut 1347.

\section{RÉSUMÉS}

La pratique des incantations, dans le cadre de la médecine populaire, était encore bien vivante et répandue en Carélie au XIX ${ }^{\mathrm{e}}$ siècle. Dans cet article, j'examine cette pratique dans son contexte chrétien. L'analyse d'un motif métapoétique et métapragmatique particulier - En puhu omalla suulla, «Je ne parle pas avec ma propre bouche»- permet de montrer la manière dont l'incantateur perçoit ses propres activités, en l'absence d'informations plus détaillées sur le contexte de performance. J'examine donc comment l'incantation construit un espace rituel particulier, dans lequel la relation entre le corps du guérisseur et celui du patient change de niveau, passe du niveau physique au niveau symbolique. Les deux différents niveaux de réalité se mêlent, et permettent ainsi de construire le cadre dans lequel la guérison devient possible. Les mots ont ainsi un pouvoir en tant qu'ils sont la condition de possibilité du rituel de guérison, et non parce qu'ils contiennent une force en eux-mêmes.

In this article I aim to examine the incantation tradition in Karelia through the prism of popular Orthodoxy. I look at a specific metapoetic motif, En puhu omalla suulla, "I do not speak with my own mouth", in which the charmer uses the divine authority to make his incantations effective. I 
aim to elucidate how the incantations construct a special ritualistic space in which the relationship between the bodies of the healer and the patient are transformed and go onto another level of reality. Additionally, I examine how the Christian vocabulary and conceptions are prevalent in this process.

Tämä artikkeli pyrkii tutkimaan Pohjois-Karjalan ja Laatokka-Karjalan loitsuperintöä kristillisenä perinteenä. Tarkastelen erityisesti yhtä motiivia jolla on tärkeä metapoeettinen ja jopa metapragmaattinen funktio, "En puhu omalla suulla". Tietäjä kertoo loitsuissa hänen teoistaan, hän kommentoi samalla kun hän toimii ja koskee potilaan vartaloa. Tavoitteena on ymmärtää kuinka tietäjä esittää verbaalisesti puheen ja sanojen voimaa. Sanojen tehokkuus johtunee siitä, että tietäjä konstruoi niiden avulla erikoisen rituaalisen tilan, jossa maailman eri ulottuvuudet sekoittuvat yhteen. Sanat luovat uuden kontekstin, missä parantajan ja potilaan kehojen välisellä suhteella on toinen merkitys: siten parantaminen on mahdollista.

\section{INDEX}

Index géographique : Carélie, Carélie du Nord, Carélie du Ladoga, Finlande, Novgorod, Russie, Salmi, Sibérie, Suède, Turku

Mots-clés : christianisme populaire, incantations, médecine populaire, poésie orale, pouvoir performatif de la parole, rituel de guérison

Thèmes : études de folklore, ethnologie, littératures orales

motsclesru НАРОДНАЯ МЕДИЦИНА, НАРОДНОЕ ХРИСТИАНСТВО, ПРИЧИТАНИЯ, СИЛА СЛОВА, ЦЕЛИТЕЛЬНЫЕ ОБРЯДЫ

motscleset loitsud, rahvakristlus, rahvameditsiin, ravimisrituaal, sõnavägi

Keywords : charms, folk Christianity, folk medicine, incantations, healing ritual, word power disciplines finnois, vepse 